\title{
Fournier Gangrene in the 75 year old Patient: A Case Report
}

\section{Nuhi Arslani ${ }^{1}$, Basri Lenjanji ${ }^{2}$}

\begin{abstract}
Aim: The aim of this article is to describe an example of the sick patient with Fournier gangrene and demonstrate the process of his treatment and the solving of problems that had incurred.

Case report: The patient is a 70-year-old man who was hospitalized for Fournier gangrene in the perineal region. We performed radical necrectomy, drainage of perianal abscess, and transversostomy. Based on wound culture we prescribed antibiotics Amoksiklav and Ciprobay. Later there was a complication in the form of a decompensated adhesion ileus, which required a re-operative intervention.

Conclusion: Fournier gangrene is a rare disease that usually affects males. The disease starts with swelling, cellulitis,higher temperature and odor. Our example describes the successful treatment of the disease with emergency surgery and re-operative intervention.
\end{abstract}

Keywords: necrotizing fasciitis, necrectomy, decompensated adhesion ileus, laparotomy

${ }^{*}$ Corresponding author: Nuhi Arslani

$\equiv \triangle$ Email: arslani.nuhi@gmail.com

1Department of General and Abdominal Surgery, University Medical Centre Maribor, Faculty of Medicine, University of Maribo, Slovenia

${ }^{2}$ Hospital and University Clinic of Kosova, Emergency Clinic,Pristina, Kosova 


\section{Full Text}

\section{Introduction}

Necrotizing fasciitis was first described in 1764 by Baurienna. It was named after Fournier, which in 1883 reported the case of five men with this disease. Fournier gangrene is a necrotizing fasciitis, which affects the subcutaneous tissue of the perineum and genitals. The disease is usually caused by an infection of the colon, the rectum, the lower part of the genito-urinary tract, or cutaneous infection of genitalia, perineum and the anus. The infection is caused by aerobes or anaerobes. Microorganisms that are usually isolated are Escherichia coli, Enterococci, Staphylococci, Streptococci, Bacterioides fragilis and Pseudomonas aeruginosa. Reasons for infection that causes the disease are superficial damage, genito-urinary diseases or colorectal diseases, they can create points of entry such as genitourinary sepsis, sepsis of the prostate, perianal sepsis and ischiorectal abscesses. There are also conditions that play an important role in the development of the disease by causing the malfunction of the immune system. The most commonly described are diabetes, obesity, alcoholism, malignancy and immunosuppression. Signs of the disease are swelling, cellulitis, necrosis, crepitation, higher temperature and odor. Disease is treated surgically with radical necrectomy of all necrotic and infected tissue as well as administartion of broad spectrum antibiotics. The mortality rate is close to $50 \%$.

\section{Case report}

The present case report is about 75-year-old man who was admitted to our hospital due to inflammation in the gluteal region. His medical history revealed that he had hemorrhoids for more than 30 years. In the last three years he has had problems with the discharge of liquid stool mixed with mucus. His symptoms had started one week prior to the admittance. The symptoms were induration in the right gluteal region, which had grown and expanded in the left gluteal region. He also complained severe pain, fever and uncontrolled leakage of stool. At admission the patient was slightly affected by the pain, he had a pulse $91 / \mathrm{min}$, arterial pressure was $126 / 69$, and body temperature was $36.6^{\circ} \mathrm{C}$. A physical examination revealed visible necrotic area of the left and right gluteal region (figure 1). There was fluctuation by palpation.

A CT was made which revealed visible infrarenal aneurysm and aneurysm of the right common iliac artery. On the same day the patient was treated with wide debridement of devitalized tissue, perianal abscess drainage and transversostomy (figure 2 and 3). The patient returned to the operating room 11 days after his first radical necrectomy for further transanal drainage. Two days later there was a complication in the form of decompensated adhesion ileus. We performed a laparotomy. The stomach contained 2L of liquid, which we aspirated. Then we saw that the thin intestinal convolutions were affixed in three places in the region of the left inguinal canal, in proximity of sigma. After we performed adhesiolysis at all three sites we placed stitches, then we made substantial lavage of the entire abdomen and dried it. 
After that we performed appendectomy and drained abdomen. We closed the incision with a running suture. At the end of the operation we concentrated on the perianal region. There we performed necrectomy and replaced bandages. Approximately one month after, the patient was discharged home (figure 4). Wound culture,

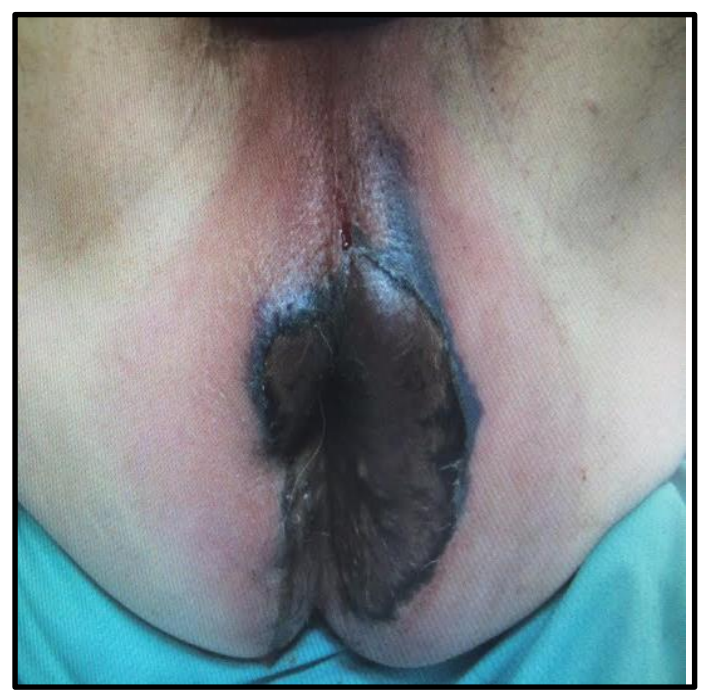

Figure 1: Fournier gangrene (necrotizing fasciitis perinanal)

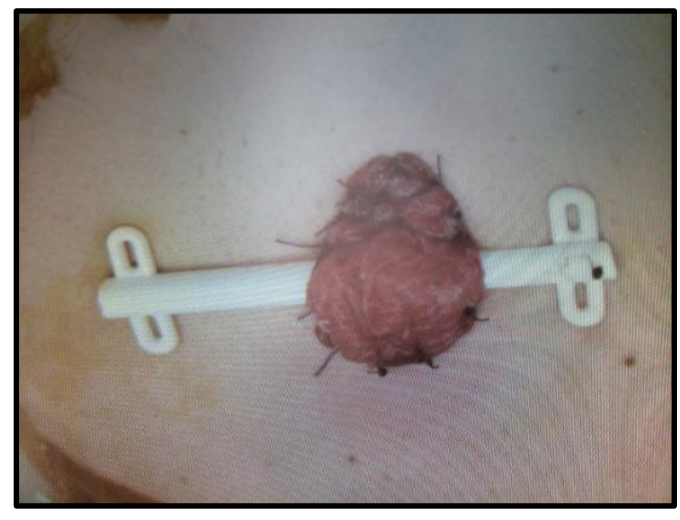

Figure 3: Derivation of protective bipolary colostomy which was taken on the day following the first operation showed E. coli, Morganella morgani, Streptococcus anginosus, Enterococcus faecalis and several anaerobes, including at least one resistant to clindamycin. Based on the wound culture we prescribed treatment with an amoxiclav and ciprofloxacin.

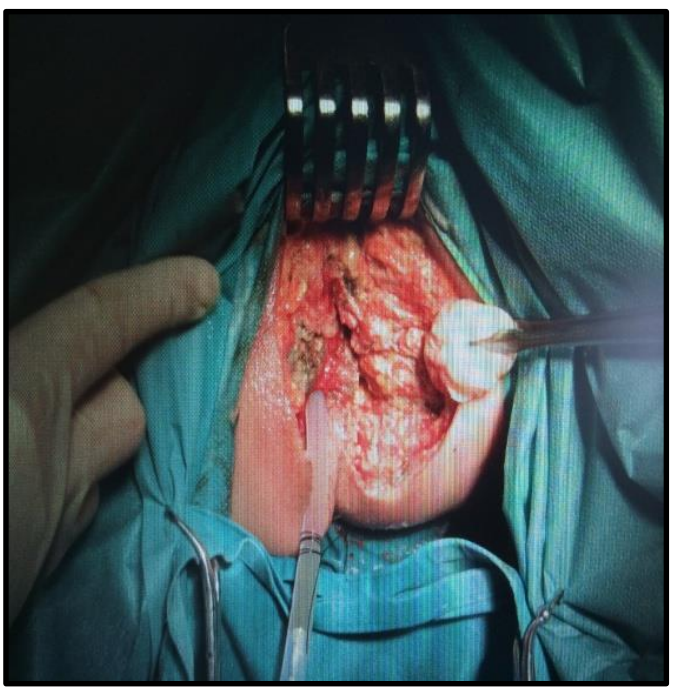

Figure 2: Immediately after the perineal radical excision

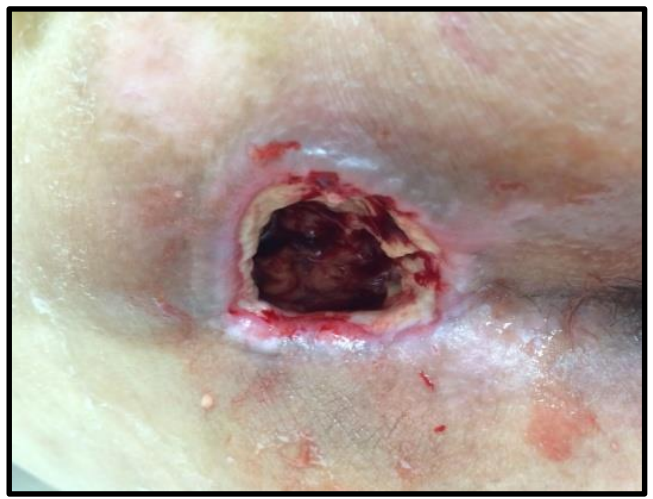

Figure 4: Wound healing per secundam intetionem one month after radical excision 


\section{Discussion}

Fournier gangrene is a form of necrotizing fasciitis. In most cases, it affects men over 50 years of age. The causes of infection that produces the disease is among others the already existing colorectal diseases. Points of entry for infects may be cutaneous injury, urogenital sepsis or perianal sepsis. Infects are normally aerobic and anaerobic bacteria, which in tissue act synergistically. Microorganism that is most frequently isolated from caused by hemorrhoids. Clinical signs of Fournier gangrene are usually intense pain, fever and swelling. Treatment of Fournier gangrene involves the application of broad-spectrum antibiotics, supportive care in the case of multiple organ failure and emergency surgery in the form of wide excision with one or more debridement. The objective is to remove the debridement of necrotic tissue, until only healthy tissue remains. The patient is usually discharged from the hospital one month after surgery. Most common isolated microorganism in wound culture is E. Coli, which was also isolated in our case. The point of entry in our case was perianal sepsis.

\section{Conclusion}

Fournier gangrene is a rare disease with high mortality. Our example describes the successful treatment of the disease with emergency surgery in which we carried out radical necrectomy of soft tissue, and transversostomy.
It also describes the resolution of the early postoperative complications.

\section{References}

1. Efem S.E.E. The features and aetiology of Fournier's gangrene. Postgrad Med J (1994); 70: $568-571$

2. Townsend, Beauchamp, Evers, Mattox, Sebastion text book of surgery,Philadelphia, Elsevier Saunders,2004, chapter 76, Aria F. Olumi Jerome P. Richie ,p. 2297

3. Erikoglu M, Tavli S, Turk S. Fournier's gangrene after renal transplantation. Nephrol Dial Transplant (2005) 20: 449-450

4. Leung E, McArdle K, Yazbek-Hanna M. Pus swabs in incision and drainage of perianal abscesses: what is the point?. WorldJ surg. 2009 Nov;33(11):2448-51

5. Vanden Bempt I, Van Trappen S, Cleenwerck I, Van De Vyvere M, De Vos P, Camps K, Celens A. Actinobaculum schaalii Causing Fournier's Gangrene. Journal of clinical microbiology, June 2011, p. 2369-2371

6. Altarac S, Katušin D, Crnica S, Papeš D, Rajković Z, Arslani N. Fournier's gangrene: etiology and outcome analysis of 41 patients. Urol Int. 2012;88(3):289-93.

7. Matilsky D, Lewiss R. E, Whalen M, Saul T. Fournier's Gangrene. Case report. Med Ultrason 2014, Vol. 16, no. 3, 262-263 